\title{
La Féminité Vue Par Simone De Beauvoir : Une Lecture Du Deuxième Sexe
}

\author{
Chioma Faith Uzoho \\ DOI: http://dx.doi.org/10.4314/ujah.v14i1.1
}

\section{Résumé}

Simone de Beauvoir est une féministe et philosophe française dont la théorie féministe et idéologie philosophique ont beaucoup marqué le 20e siècle. Elle fait à travers son œuvre théorique, Le deuxième sexe publiée en 1949, une dénonciation très rigoureuse de l'infériorisation et de l'assujettissement de l'être féminin (la femme). On ne peut pas aujourd'hui entreprendre une étude féministe sérieuse sans faire assez de référence à cette philosophe existentialiste connue comme la mère du féminisme occidental. En plus, elle est la plus grande théoricienne du féminisme occidental. Il s'agit dans cet article d'un examen critique de la vision beauvoirienne de la féminité à la lumière de son ouvre théorique, Le deuxième sexe. Ceci rendra plus accessibles les discussions sur Beauvoir.

\section{Abstract}

Simone de Beauvoir is a French feminist philosopher, whose feminist theory and philosophical ideology largely marked the 20th century. Through her theoretical work, Le deuxième sexe published in 1949, she does a very firm denunciation of the disparagement and subjugation of women. One cannot undertake today a serious feminist study without making enough reference to this existentialist philosopher, widely known as the mother of Western feminism. In addition, she is the greatest Western feminist theoretician. This article is a critical examination of the beauvoirian conception of 
femininity in the light of her theoretical work, Le deuxieme sexe. This no doubt will make discussions on Beauvoir much more accessible.

\section{Introduction}

Simone de Beauvoir développe très tôt (à quinze ans) dans sa vie, une passion très brulante pour la littérature. Elle conçoit le métier d'un écrivain comme celui qui doit toucher des aspects importants de sa société. Elle sera politiquement et philosophiquement engagée prenant position avec Sartre dans sa politique de gauche et dans sa philosophie existentialiste. C'est ainsi qu'elle fait preuve d'un féminisme qui penche sur l'idéologie existentialiste qu'il n'existe pas de nature humaine. Elle conçoit ainsi la féminité comme une idée complètement sociale et non jamais naturelle. Elle devient la figure de proue du féminisme en décrivant une société qui maintient la femme dans une situation d'infériorité. Elle fait alors une analyse de la condition féminine à travers les mythes, les civilisations, les religions, l'anatomie et les traditions. Elle défend la maternité facultative et l'avortement. Le mariage pour elle, met la femme dans une condition de dominance par son mari ; une condition d'esclavage dont elle n'arrivera pas à se libérer.

\section{Le statut de la femme chez Beauvoir}

La relation 'amoureuse' de Simone de Beauvoir avec son compagnon de vie, Jean-Paul Sartre témoigne beaucoup de sa conception de la femme en tant qu'un être qui est censé être aussi libre que l'homme. C'était une relation marquée immensément par l'infidélité extrême, l'inceste et toutes sortes de perversions sexuelles tout au nom de la liberté absolue. Tout comme son ami, Beauvoir avait de jeunes amants et également des adolescentes parmi lesquelles il y a ses élèves, Olga et Nathalie Sorokine avec qui elle a eu des relations charnelles. Marie Couillard nous indique dans son article en ligne, 'La lesbienne selon Simone de Beauvoir et Nicole 
Brossard : identité ou figure convergente ?', que 'Simone de Beauvoir consacre environ trois pourcent de son ouvrage à la lesbienne' (n.pag).

Dans le chapitre sur 'la lesbienne', Beauvoir soutient que le lesbianisme, tout comme l'hétérosexualité, peut être vécu de mauvaise foi ou de manière authentique, selon la façon dont on s'exerce le choix. Cela veut dire que malgré l'importance du corps, il ne doit pas déterminer la femme comme individu. La femme, en tant qu'un être libre, doit, selon la conception beauvoirienne de la féminité, reconnaître le fait qu'elle est absolument libre de choisir ce qu'elle fait avec son corps. C'est seulement quand la femme n'est pas indépendante qu'elle devient l'Autre pour l'homme, c'est-àdire, un être inférieur fait pour être possédé comme un objet.

Ce n'est que dans cette situation qu'elle se transforme en une personnalité passive et immanente. Seule cette situation, d'après elle, peut transformer les petites filles en 'femmes féminines' sous la pression des parents et de la société. Elle voit cette réaction comme celle qui peut être le fruit d'une éducation et non pas une réaction due à une nature quelconque. Par là, elle insiste qu'il n'existe aucun facteur naturel à la féminité.

Le deuxième tome du Deuxième sexe de Beauvoir s'étend sur les conditions sociales et culturelles de son époque. Ici, elle décrit la femme telle qu'elle est en expliquant que tout dépend du sens qu'on donne au verbe 'être'. Elle remarque:

C'est la portée du mot être qu'il faudrait s'étendre ; la mauvaise foi consiste à lui donner une valeur substantielle alors qu'il a le sens dynamique ... être c'est être devenu, c'est avoir été fait tel qu'on se manifeste (25).

Autrement dit, rien de ce qui caractérise la femme à présent ne laisse présumer de ce qu'elle sera demain. 
Bien que Beauvoir ait consacré une partie de son essai à parler du lesbianisme, sa conception du sujet reste floue parce qu'elle n'aborde pas l'aspect charnel de ses relations avec les femmes, malgré le fait qu'elle semble motivée par la volonté de tout dire et d'être sincère. Néanmoins, il y a des témoignages dans sa vie qui sont la preuve de sa position. Par exemple, pendant l'Occupation allemande de la France en 1943, elle a été congédiée de sa carrière de professeur pour un 'détournement de mineure' suite aux accusations portées contre elle par la mère d'une de ses élèves, Sorokine. Beauvoir ne s'est pas ouvertement déclarée lesbienne mais elle a eu un bon nombre de liaisons amoureuses et sexuelles avec des femmes pendant sa jeunesse et vers la fin de ses jours; cette réalité a été pourtant découverte dans son journal intime après sa mort. Dans son article en ligne intitulé 'la lesbienne dans $L e$ deuxième sexe de Simone de Beauvoir', Marie-Jo Bonnet cite les mots de Simone de Beauvoir dans sa lettre à Sartre en 1939 :

Ça me fait quand même drôle d'être passionnément aimée de cette manière féminine et organique par deux personnes: Védrine et Sorokine ... On a eu des étreintes passionnées, et à vrai dire j'ai pris quelque goût à ces rapports.

De Beauvoir cédera bientôt aux avances de Sartre qui couche aussi avec Arlette, sa fille adoptive. Le couple partage aussi l'amour de Bianca (16ans), la sœur cadette d'Olga. Au début des années 1980 aux Etats-Unis, quelques philosophes féministes telles, Anne Ferguson, Claudia Gard et Marilyn Frye dans leurs études critiques sur Le deuxième sexe avaient reproché à Simone de Beauvoir d'avoir nié son identité lesbienne tandis qu'elle semblait maintenir une distinction entre la lesbienne et la femme. (Ferguson : 289, Frye : 300, Gard : 290). 
De Beauvoir conçoit le métier d'un écrivain comme celui de quelqu'un qui doit beaucoup toucher à la vie de la société. Elle affirme dans La force de l'âge que 'la littérature apparaît quand quelque chose dans la vie se dérègle' (532).

C'est-à-dire que l'écrivain est plus ou moins un trompettiste qui doit lancer un appel de clairon pour attirer l'attention du monde chaque fois qu'il y a une anomalie dans la société. C'est ainsi qu'elle écrit Le deuxième sexe où elle fait une sorte d'analyse de toutes formes d'assujettissement dont la femme a été l'objet pendant son époque. Dans le texte, Simone de Beauvoir s'interroge sur la définition de la femme. Est-il vrai que la femme se définit relativement à l'homme ? Est-ce que la femme existe pour et par le sexe masculin? Elle examine avec rigueur, le sort réservé à la femme. Elle affirme qu'il n'y a pas de différences à priori entre les sexes et insiste qu'au fil de l'histoire, le problème de la femme a toujours été l'homme.

L'émancipation féminine d'après Beauvoir, suppose une révolte des exploitées du travail et du sexe. Pour soutenir son argument, elle emploie trois exemples : les Américains colonisés par les Anglais pendant le $18^{\mathrm{e}}$ siècle, les esclaves noirs des Etats-Unis et Le Prolétariat. Ce sont surtout des groupes qui n'ont pas été en mesure de se développer puisqu'ils étaient terriblement assujettis. Beauvoir énonce à partir de ces exemples, une loi générale sur les conditions de la création, les faits historiques et sociologiques.

Le deuxième sexe de Simone de Beauvoir a été beaucoup influencé et illustré par sa relation anticonformiste avec Jean-Paul Sartre dont l'idéologie de la liberté a fourni et continue de fournir, même de nos jours, les fondements initiaux de la théorie féministe de la liberté. C'est grâce à cette 
relation qu'elle est connue comme une femme indépendante et totalement libérée.

Beauvoir est d'une famille catholique. Sa famille a essayé de l'éduquer dans de bonnes manières féminines de son époque, mais elle rejette très tôt dans sa vie, les enseignements religieux et conventionnels en se déclarant athée. Elle a manifesté assez tôt dans sa vie, les traits de la masculinité. Le deuxième sexe, souvent considéré comme la 'Bible du féminisme' est très proche de l'existentialisme athée non seulement car son auteur est athée mais il y a aussi le fait qu'elle considère la féminité, non comme un fait naturel, mais comme un fait tout à fait social dans la mesure où, pour elle, il n'existe pas de nature féminine. Si les existentialistes déclarent que l'existence de l'homme précède son essence, il s'en suit alors que l'existence de la femme précède son essence, ce qui est pour elle, la preuve que la femme n'a pas de nature innée. L'homme existe d'abord, après, il se définit; dans la même mesure, la femme existe d'abord et se définit après. Si l'existentialisme préconise que l'homme en tant qu'être pensant est absolument libre de choisir et de déterminer son essence, la même chose doit s'appliquer à la femme. Si l'existentialisme prêche que l'existence de l'être humain précède son essence, cela signifie pour Beauvoir qu'il n'existe pas de nature féminine. C'est ainsi que Beauvoir conçoit plutôt la féminité comme un produit social et non pas issue d'une nature innée.

Par son essai, Beauvoir met au jour, les conditions physiologiques et sociales de la femme : les règles qui font honte, les grossesses subies, le travail domestique infiniment répété (68). Elle considère la femme comme un être qui doit acquérir une indépendance totale et vivre libre et autonome (31). Elle insiste que la consécration totale de la femme à son mariage et à ses enfants limite beaucoup sa liberté. C'est une situation qui d'après Beauvoir, vient du fait que la femme ne se sent pas capable de rester célibataire surtout pour des 
raisons sociales: la société voit la femme comme celle qui est inférieure à l'homme; elle doit alors avoir un mari auquel elle doit rendre des comptes. Beauvoir, qui tire l'inspiration du Deuxième sexe de l'idéologie libératrice de Sartre, insiste que le rôle réservé à la femme dans la famille contribue immensément au fait qu'elle arrive rarement à se réaliser. Elle souligne profondément les notions de domination et d'aliénation qu'elle emploie largement dans ses analyses.

Le deuxième sexe est souvent employé comme référence dans des discours féministes. Bien que l'essai soit considéré comme la 'Bible du féminisme', Beauvoir avait nié une prise de position féministe après sa publication. Elle affirme ainsi dans La force de l'âge :

Je n'ai jamais nourri l'illusion de transformer la condition féminine, elle dépend de l'avenir du travail dans le monde, elle ne changera sérieusement qu'au prix d'un bouleversement de la production. C'est pourquoi j'ai évité de m'enfermer dans ce qu'on appelle 'le féminisme ... si mon livre a aidé les femmes, c'est qu'il les exprimait et réciproquement, elles lui ont conféré sa vérité (267).

Elle avait tout d'abord conçu l'essai comme une étude théorique ou plutôt un essai philosophique. Mais elle changera plus tard d'avis en expliquant que le changement d'avis est dû à sa reconnaissance $d u$ fait que le socialisme ne peut certainement pas aboutir à l'émancipation de la femme. Elle explique, lors d'une entrevue par Alice Jardine en 1979, que 'the emancipation of women must be the work of women themselves, independent of the class struggle' (235). Beauvoir insiste non seulement sur l'amélioration de la condition de la femme, mais elle maintient que le système qui provoque 
l'inégalité du sexe ainsi que l'injustice soit entièrement détruit. Dans son article, 'Les femmes s'entêtent', elle écrit :

... Il m'a semblé longtemps que certains inconvénients inhérents à la condition féminine devraient être simplement ... surmontés, ... En fait, accepter entre les deux sexes la moindre inégalité, c'est consentir à l'inégalité (250).

D'après Albistur et Armogathe, 'il ne faut pas avoir peur que tout le féminisme contemporain procède du Deuxième sexe' (606).

De même, Yvette Roudy affirme:

S'il n'y avait pas eu l'analyse théorique, historique, très complète, très solide et demeurée vraie de Simone de Beauvoir, les effets des mouvements féministes nés autour de 1968 n'auraient pas été aussi puissants (113).

Beauvoir elle-même affirme dans un film produit par Josée Dayan et Malka Ribowska que, 'ce livre peut avoir une certaine valeur parce qu'elles (les féministes) n'ont pas tellement de théoriciennes' (68). C'est ce qu'affirme Rémy lorsqu'il écrit que Le deuxième sexe a donné à la lutte des femmes le substrat théorique qui lui manquait (29). Beauvoir voit ainsi l'essai comme une œuvre théorique très convenable pour les féministes modernes.

Plusieurs féministes des années 1970s vont plus tard adopter des idées développées dans l'œuvre de Beauvoir, à savoir, le refus du mythe culturel, la question du mariage, la famille et la maternité, l'ennui de rester femme au foyer, la dépendance économique des femmes mariées. Ces féministes revendiquent le droit de la maternité volontaire, voire, le droit d'exister sans être mère ; elles rejettent entièrement le modèle de la bonne mère au foyer. Dans un article, 'Maternité', Laborie et Collins affirment que la maternité implique non seulement 'le pouvoir de donner une vie, mais 
une fonction sociale au nom de laquelle revendiquer des droits politiques ou des droits sociaux ...' (96).

Les revendications de ces féministes sont formulées essentiellement avec le seul but de faire tomber tous les obstacles qui peuvent empêcher la femme de se réaliser et d'accéder au même titre que l'homme. Firestone identifie la maternité, la famille et le mariage (dans la formule de Beauvoir) comme institutions du système patriarcal qui font naître l'oppression sexuelle, socio-économique ou politique des femmes (56). D'autres féministes, encore influencées par l'essai de Beauvoir, dénoncent la maternité comme vocation naturelle de la femme puisque cela la force à accepter des conditions aliénantes qui l'empêchent de vivre une vie autonome tout en l'enfermant dans des rapports de dépendance économique et affective.

Le deuxième sexe semble répondre au désir de Sartre d'une littérature qui dévoile la société en agissant sur le monde. L'essai de Beauvoir reste jusqu'à nos jours, l'étude fondamentale sur la situation de la femme. Il reste le fondement du féminisme égalitaire dans le monde occidental. Pour la plupart des féministes contemporains, l'importance de cet essai en tant qu'œuvre fondatrice ne fait pas de doute. Savigneau observe à propos de l'essai que '... il s'agit là d'un énorme travail, d'une considérable enquête minutieuse, structurée, pensée ... vers la libération' (30). Dans son œuvre, Simone de Beauvoir, conflits d'une intellectuelle, Toril Moi remarque que

Bien avant l'apparition du mouvement de femmes, elle (de Beauvoir) a relevé dans Le deuxième sexe, toutes les questions que les féministes d'aujourd'hui s'efforcent de résoudre. Ce livre a littéralement transformé l'existence de milliers de femmes: je ne vois 
aucun livre qui ait eu un tel impact au cours de ce siècle (5).

A travers Le deuxième sexe, Beauvoir soutient dans la perspective de l'existentialisme athée qu'il n'existe aucune nature des femmes, mais que la féminité est un produit social. L'idée centrale du livre, 'on ne naît pas femme, on le devient' (13) présuppose l'argument de Sartre que 'l'existence précède l'essence'. C'est-à-dire que si l'homme (l'être humain) n'a pas de nature préexistante, la même théorie s'applique à la femme. Cela signifie non seulement qu'il n'y a pas de nature féminine préétablie qui justifierait la ségrégation sexuelle, mais que la condition de la femme et l'existence en tant que femme est un combat.

A part l'inspiration que Beauvoir a tirée de l'idéologie sartrienne, elle a aussi été influencée par son environnement social. Elle est d'une famille bourgeoise aisée, d'une couche sociale où les femmes étaient beaucoup marginalisées : elles ne travaillaient pas et n'étaient pas alors salariées. Beauvoir se révolte tôt contre cette condition et exerce un métier pour gagner sa vie. Elle trace, à travers Le deuxième sexe, l'origine de l'aliénation et de l'infériorisation de la femme dans la société en général et dans presque tous les domaines hors du foyer familial. Elle voit la femme comme celle qui est la plus 'profondément aliénée' (70) de toutes les femelles mammifères. Cela explique pourquoi elle est celle qui refuse, d'après Beauvoir, le plus violemment cette aliénation (70).

De Beauvoir proteste contre la vue conventionnelle que la femme doit se consacrer entièrement à son mariage et à ses enfants alors que l'homme 'dirige le monde'. Pour elle, c'est une position qui limite beaucoup la liberté de la femme.

En ce qui concerne la dite infériorité de la femme, elle insiste que ' ... ce n'est pas l'infériorité des femmes qui a déterminé leur insignifiance historique: c'est leur insignifiance qui les a vouées à l'infériorité' (225). Qu'une femme soit mère, épouse ou fille, elle ne se définit qu'en 
fonction de l'homme et jamais pour elle-même. La femme incarne toujours '1'Autre' et sacrifie, la plupart du temps, sa carrière pour celle de son mari. Pour Beauvoir, la femme ne doit pas abandonner sa carrière pour son mari et ses enfants. En parlant de la situation globale de la femme, elle insiste que l'homme et la femme sont responsables de cette situation et que l'homme ne devrait pas encourager la femme à abandonner sa carrière. Elle explique de plus que dans un monde où les deux sexes seraient égaux, les deux seraient plus libres.

Du point de vue historique et mythique, puis, en s'appuyant sur des expériences vécues, Beauvoir montre comment, d'une manière ou d'une autre, la femme reste toujours l'esclave de l'homme. Dans son analyse biologique de la condition féminine, elle constate que la vie de la femme est pleine de crises. Elle parle ainsi de ' ... crise de puberté et de la ménopause, 'malédiction' mensuelle, grossesse longue et souvent difficile, accouchement douloureux et parfois dangereux (qui) sont caractéristiques de la femelle humaine' (70), par rapport à la 'vie génitale (de l'homme) qui se déroule d'une manière continue, sans crises et généralement sans accident' (71).

Bien que Beauvoir croie que ces données biologiques sont d'une importance extrême et jouent dans l'histoire de la femme, un rôle de premier plan, elle affirme qu'elles ne constituent pas pour elle, un destin figé (71). Ces données biologiques pour elle, n'expliquent pas pourquoi la femme est ' 1 'Autre' et ne suffisent pas non plus pour justifier qu'elle assume un rôle subordonné. Après tout, 'ce n'est pas la nature qui définit la femme : c'est celle-ci qui se définit en reprenant la nature à son compte dans son affectivité' (78).

Un grand nombre de personnages féminins dans la fiction de Beauvoir sont présentés comme étant brisés et 
malheureux. Ce sont des femmes dont l'existence diffère immensément de celle des personnages masculins. Les femmes se sacrifient au nom de l'amour, un trait qu'on ne voit pas chez les personnages masculins. En parlant de l'amour total chez ses personnages féminins, Beauvoir explique, lors d'une interview par Bernard Frechtman, que les femmes, plus que les hommes, ont la capacité de se montrer compatissantes. On peut pourtant voir cette affirmation comme une contradiction de sa fameuse déclaration qu' 'on ne naît pas femme ...' (13).

A l'encontre de la situation 'idéale' que désire Beauvoir, la femme ne se définit qu'en fonction de l'homme et jamais pour elle-même puisqu'elle incarne 'l'Autre', ce qui explique l'impossibilité de relations de réciprocité et d'égalité entre les hommes et les femmes. Il y a dans l'essai, la contestation d'une nature féminine. Pour elle, l'aliénation et l'infériorisation de la femme ne sont pas du tout liées à la nature même de la femme, mais résultent de circonstances historiques et sociales. Elle insiste sur la nécessité pour la femme d'assumer elle-même son existence. Rien n'empêche la femme d'accéder comme tout 'sujet' (être pensant) à une libre transcendance qui s'exprimerait par une activité constructive (ce qui sera l'équivalent de l'acte authentique chez Sartre).

Beauvoir nous signale l'importance d'exercer un métier; elle est d'avis que c'est à travers le métier que la femme peut conquérir sa dignité d'être humain. L'homme ne doit pas continuer à concevoir la femme comme 'objet', c'est-à-dire, celle qui est faite pour certaines tâches proprement féminines. Elle voit le rapport homme-femme comme étant le même que le rapport capitaliste-ouvrier ou colon-indigène.

Nous notons que l'argument soulevé par Beauvoir que la femme n'est pas douée d'une nature spéciale s'oppose complètement à la conception de la signification de l'être 
féminin par Dieu, le Fabricateur de la femme, qui, dès le début, nous signale que la femme a une ' ... nature plus délicate' (1 Pierre, $3: 7$ ) et doit être traitée par l'homme avec tant de respect. Il a dans la même mesure défini précisément les rôles qu'il a assignés à cet être délicat: ceux de l'enfantement et de la soumission envers le mari (1 Pierre, $3: 1)$. Par ce rôle, la nature a déjà doté à la femme, le statut de l'élément constitutif de la famille. Non seulement qu'elle assume l'accouchement de l'enfant mais c'est elle qui assure l'éducation informelle de ce dernier. Cela ne veut pas dire que la femme doit se métamorphoser en 'objet' pour être entièrement possédée par l'homme. Elle doit tout simplement se soumettre à son mari (Ephésiens, 5 :22-23) qui à son tour, devrait l'aimer de sorte qu'il serait même prêt à se sacrifier pour elle (Ephésiens, $5: 25$ ).

Notons aussi que le fait que le développement physiologique de l'être féminin, dès la puberté, est un peu plus compliqué par rapport au mâle démontre davantage la délicatesse de la nature féminine. De plus, on ne peut pas ignorer les fonctions biologiques de la femme, telles que l'enfantement et l'allaitement; ce sont des qualités et des rôles naturellement féminins et rien d'autre parce qu'il est complètement impossible de voir les hommes accomplir ces fonctions. On ne peut pas alors réfuter cette vérité qu'il existe une nature féminine ainsi qu'une nature masculine.

Bien qu'il existe ces différentiations naturelles, cela ne justifie pas la conception traditionnelle du corps féminin comme 'objet' de plaisir et une machine à fabriquer des enfants. Le fait de ramener le corps féminin à un corps-objet du plaisir de l'homme est abusif et inhumain. C'est cette idée de la femme-objet que doit combattre le féminisme. C'est-àdire, celle de redonner à la femme, une identité propre par 
rapport à l'homme et de lui restituer le statut d'un être respectable.

Il y a dans Le deuxième sexe, une critique amère de la religion chrétienne que Beauvoir croit contribuer à l'oppression de la femme. Elle insiste que la position assignée à la femme dans la Bible est presque la même avec celle qu'occupent les lépreux. Elle proclame alors qu'

A travers Saint Paul s'affirme la tradition juive, farouchement antiféministe. Saint Paul commande aux femmes l'effacement et la retenue,...fonde sur l'Ancien et le Nouveau Testament le principe de la subordination de la femme à l'homme. (Elle cite) 'L'homme n'a pas été tiré de la femme, mais la femme de l'homme; et l'homme n'a pas été créé en vue de la femme, mais la femme en vue de l'homme'. Et ailleurs: comme l'Eglise est soumise au Christ, ainsi soient soumises en toutes choses les femmes à leurs maris (157).

Beauvoir n'a pas bien exploité cette idée de soumission car la même Bible ordonne qu'on (homme et femme) se soumette l'un à l'autre (Ephésiens, $5: 21$ ). Ce que la religion chrétienne recommande donc, c'est une relation réciproque bien que la femme doive reconnaitre le fait que le mari occupe plus ou moins la position de Christ par rapport à l'Eglise. Il est ainsi exigé que l'homme aime sa femme de sorte qu'il doive être prêt à se sacrifier tout comme le Christ a aimé l'Eglise et s'est sacrifié à elle (Ephésiens, 5 :25). Notons aussi que le fait que la soumission est exigée de la femme auprès de son mari n'implique pas qu'elle doit occuper la même place qu'un esclave. En réalité, la relation qui doit exister entre le mari et la femme est tout simplement comparable à celle qui existe entre le Christ et l'Eglise.

Du point de vue de la nature de la femme, Rousseau n'est pas du côté de Beauvoir, il est plutôt du côté de Sartre 
car il voit la femme comme un être qui est naturellement dotée de la passivité. Sa position sociale, d'après Rousseau, ne serait pas alors la même que celle de l'homme.

Il est pertinent de remarquer ici que dans les années 1970 se développe une forme de féminisme qui s'oppose au féminisme de Simone de Beauvoir. Un groupe féministe s'est mis à affirmer précisément ce qu'avait nié Beauvoir. Pour ce groupe, il y a une nature féminine qui implique forcément une essence féminine, Mais ils soutiennent que cette nature n'est plus définie par les hommes, mais par les femmes elles-mêmes. Un des précurseurs de ce courant, Ingrid Galster, affirme en imitant la formule fameuse de Beauvoir : 'on naît fille ou garçon, et la physiologie est un destin pour la fille ou le garçon' (591). Cela signifie qu'il existe une nature féminine qui est issue de la physiologie. Du moins, le fait que le développement physiologique de l'être féminin est beaucoup plus compliqué que celui du mâle est une preuve de la délicatesse de son être. Il existe sans doute une nature féminine.

De Beauvoir conçoit l'existentialisme comme une philosophie absolument athée. Cela explique pourquoi elle ne croit pas à la nature féminine car l'acceptation d'une nature féminine impliquera l'acceptation de l'existence de Dieu. Elle croit que l'être humain (y compris la femme) se trouve dans une situation qu'il cherche à dépasser dans un mouvement qu'elle appelle 'transcendance'. Pour elle, c'est l'obligation de tout être humain d'assumer le choix de soimême en liberté. Ce n'est qu'à travers une liberté consciemment vécue et réalisée que la différence humaine serait établie. Elle nous affirme à travers Le deuxième sexe qu'une femme qui se résigne à sa situation sans choisir ellemême, de façon libre et responsable, est coupable d'une faute morale. 
Contrairement à Sartre, Beauvoir prévoit le cas où l'on peut être empêché de se dépasser et contraint à rester dans l'immanence. C'est cette situation qui caractérise pour Beauvoir, la condition de la femme. D'après elle, l'homme, dès le début assume la place de 'sujet' en réservant à la femme, l'état d' 'objet'. Elle remarque que 'le rapport de la femme au mari, de la fille au père, de la sœur au frère, c'est un rapport de vassalité' (361-362). Elle assimile le rapport homme-femme au rapport capitaliste-ouvrier marqué par l'oppression. Elle insiste sur la nécessité pour la femme d'assumer elle-même son existence.

Elle cherche donc à protester contre cette différence de sexes qu'elle croit être issue des faits culturels et non issue des faits naturels. Tout comme Chaperon Sylvie qui soutient que ' ... les différences qui existent entre les sexes viennent de l'oppression subie par les femmes dans la société...'(27), Beauvoir insiste que l'aliénation et l'infériorisation de la femme sont issues des circonstances sociales et historiques et non pas issues d'une nature féminine. Rien n'empêche la femme d'accéder, comme tout 'sujet' (être pensant), à une libre transcendance qui s'exprimerait par une activité constructive.

En ce qui concerne donc la politique sexuelle chez Sartre et Beauvoir, on voit qu'il existe moins de similarités que de différences. Le point commun qui relie le couple, c'est le fait qu'ils partagent la même idéologie existentialiste soulignant surtout la liberté et l'athéisme. Dans leurs conceptions de la féminité, Sartre présente la féminité d'une manière à suggérer qu'il existe une nature féminine tandis que de Beauvoir croit que la féminité n'est qu'un produit social. Elle voit la féminité comme un 'devenir' lorsqu'elle affirme dans Le deuxième sexe qu' 'on ne naît pas femme, on le devient' (13), ce qui signifie qu'il n'existe aucune nature de la femme. 
Beauvoir voit la femme comme celle qui doit être aussi active que l'homme; pour elle, la femme doit être capable de transcender sa situation pour exercer un métier puisque c'est à travers le métier qu'elle peut conquérir sa dignité d'être humain.

\section{Conclusion}

Nous avons examiné dans cet article, la vue beauvoirienne de la féminité. En guise de conclusion, nous signalons que sa conception de la féminité est non seulement fourbe, mais blasphématoire, car elle nie le fait qu'il existe de nature féminine, mais que le rôle assigné à l'homme est complètement différent de celui assigné à la femme.

La position que nous avons prise en vue de l'argument de Simone de Beauvoir concernant la féminité peut être résumée dans les points suivants :

- Nous soutenons la position prise par le Créateur de la femme que la femme a une nature délicate, il existe alors une nature féminine. Il va de suite qu'il existe alors une nature humaine.

- Il y a naturellement des rôles assignés à la femme par sa nature, ceux de l'enfantement et de l'allaitement; l'homme aussi a ses rôles naturels.

- La position naturelle de la femme est une position de dignité, rien ne peut donc justifier l'aliénation et l'infériorisation de la femme.

- En ce qui concerne la soumission, Dieu ordonne à la femme de se soumettre à son mari.

- Dieu ordonne à l'homme d'aimer et de respecter sa femme. Selon le plan admirable de Dieu, le mari doit aider sa femme à surmonter ses faiblesses afin que tous les trésors de sa féminité puissent s'épanouir. 
- En nous basant sur les arguments ci-dessus, nous insistons qu'il doit plutôt exister entre l'homme et la femme, une relation de réciprocité puisque Dieu les a créés différents mais complémentaires, ce qui signifie que chacun ne peut pas se passer de l'autre.

Chioma Faith Uzoho is of the Department of Modern European Languages, Nnamdi Azikiwe University, Awka

\section{Quvres Citées}

Albistur, M. et Armogathe, D. Histoire du féminisme français. Paris : Editions des Femmes, 1977.

Beauvoir, Simone. La force de l'âge. Paris : Gallimard, 1986. _ _ _. Le deuxième sexe (Tome 1), Paris: Gallimard, renouvelé en 1976.

Collins, M. et Pierce, C. 'Holes and Slime: Sexism in Sartre's Psychoanalysis.' Women and Philosophy 1.1 (1976): 112-27.

Couillard, Marie. 'La lesbienne selon Simone de Beauvoir et Nicole Brossard : identité ou figure convergente ?'. Mis en ligne le 15 février, 2006. Consulté le 23 novembre, 2009. visites.unb.br/ih/labrys/les.htm.

Dayan, J. et Ribowska, M. Un film: Simone de Beauvoir, Paris : Gallimard, 1979.

Ferguson, Anne. 'Lesbien identity: Beauvoir and History.'

Hypatia Reborn:Essays in Feminist Philosophy (1990): 203-08.

Firestone, Shulamith. La dialectique du sexe. Paris: Stock, 1972.

Frye, Marilyn. History and Responsibility: Essays in Feminist Philosophy,Bloomington: Indiana University Press, 1990.

Galster, Ingrid. Position des féministes français. Metzner : Hiltrud et Renate, 1999. 
Jardine, Alice. 'Interview avec Simone de Beauvoir.'Signs 5.2 (1979) : 224-36.

La Sainte Bible. Société Biblique Française. Paris : Alliance Biblique Universelle, 1984.

Remy, M. De l'utopie à l'intégration: Histoire des mouvements de femmes. Paris : L'Harmattan, 1990.

Roudy, Yvette. A cause d'elles. Paris : Albin Michel, 1985.

Savigneau, Josyane. 'La révolution du Deuxième sexe.' Le Monde, le 19 janvier, 1999.

Toril, Moi. Simone de Beauvoir, conflits d'une intellectuelle. Paris: Editions Diderot, 1995. 